\title{
REVISÃO SISTEMÁTICA DE Tagetes minuta L. (Asteraceae): USO POPULAR, COMPOSIÇÃO QUÍMICA E ATIVIDADE BIOLÓGICA
}

Recebido: 08/12/2014

\author{
SCHIAVON, Diane Bender Almeida ${ }^{1}$; \\ SCHUCH, Luiz Filipe Damé ${ }^{1}$; \\ FACCIN, Ângela ${ }^{1}$; \\ GONÇALVES, Carolina Lambrecht ${ }^{1}$; \\ VIEIRA, Viviane Seixas Cardoso ${ }^{1}$; \\ GONÇALVES, Helena Piúma ${ }^{1}$.
}

Aceito: 02/06/2015

${ }^{1}$ Laboratório de Doenças Infecciosas, Departamento de Veterinária Preventiva, Faculdade de Veterinária, Universidade Federal de Pelotas.

\section{RESUMO}

T

agetes minuta L. é uma planta aromática vulgarmente conhecida como chinchilho, cravo-de-defunto, chinchila, picão-do-reino entre outros. O objetivo do trabalho foi executar uma revisão sistemática sobre Tagetes minuta com a finalidade de identificar os principais componentes químicos, usos populares e atividades biológicas. Como estratégia de busca da revisão sistemática, a palavra-chave "Tagetes minuta" foi utilizada no Scielo, Pubmed e Google acadêmico. Na pesquisa inicial, foram identificadas 429 publicações, sendo 384 no Google acadêmico, 9 no Scielo e 36 no Pubmed. Após a leitura do título e do resumo, foram avaliados 111 trabalhos por se enquadrarem dentro dos critérios de inclusão. Na segunda etapa, foi realizada a leitura da publicação e se estas se enquadravam nos objetivos do trabalho, a publicação era selecionada. Com este procedimento, 51 publicações foram escolhidas. A revisão sistemática mostrou que a planta Tagetes minuta, além de ser popularmente conhecida por indicações medicinais, foi cientificamente testada e apresentou atividade antibacteriana, antifúngica, larvicida, inseticida, antiparasitária, nematicida, anti-hiperglicêmica, antitumoral, biocida e antioxidante.

Palavras-chave: Tagetes minuta L. Revisão sistemática. Planta bioativa. 


\section{INTRODUÇÃO}

A Tagetes minuta L. é uma planta aromática vulgarmente conhecida como chinchilho, cravode-defunto, chinchila, picão-do-reino, rojão, cravo, coari-bravo, cravo-de-viúva, cravo-bravo, cravo-de-anjo, chilchita, chil-chil, chinchilla, manzanilla de La sierra, suico. É um subarbusto anual, ereto, pouco ramificado, com cheiro característico e de 1-2 m de altura. Possui folhas compostas e flores em pequenos capítulos amarelos. O nome "minuta" faz referência ao tamanho das flores que são muito pequenas quando comparadas com o tamanho da planta. Multiplica-se facilmente por semente, cresce naturalmente a partir da primavera e praticamente desaparece com o início do inverno. A floração ocorre principalmente no final do verão, atraindo abelhas (KRUG, 2007). O gênero Tagetes pertence à família Asteraceae, é composto por 56 espécies, sendo 27 anuais e 29 perenes (BELTRÁN, 2008; CHAMORRO et al., 2008; FERRAZ; FREITAS, 2008; HERRERA, 2002; LORENZI; MATOS, 2008; PARRAS et al., 1979; ROCA et al., 2009; SENATORE et al., 2004; VISINTIN; BERNARDELLO, 2005).

Esta planta parece ser nativa da América Central e, atualmente, ocorre espontaneamente na Bolívia, Paraguai, Argentina, México e no Brasil, onde é encontrada nos estados do Piauí, Pernambuco, Bahia, Mato Grosso do Sul, Goiás, Minas Gerais, Rio de Janeiro, São Paulo, Paraná, Santa Catarina, Rio Grande do Sul e no Distrito Federal (HATTORI, 2009; ROCA et al., 2009).

O óleo essencial de T. minuta tem larga utilização como condimento e em perfumaria, além de ser conhecido por suas propriedades biocidas. Vários estudos sobre esta planta relatam que há variações na composição do óleo essencial, de acordo com o local de colheita, a fase de crescimento, as diferentes partes da planta, além disso existem diferentes quimiotipos na espécie (CHAMORRO et al., 2008).

A revisão sistemática é uma investigação científica que tem como objetivo reunir, avaliar criticamente e conduzir uma síntese dos resultados de múltiplos estudos primários. Faz uso de uma abordagem sistemática, com metodologia claramente definida, visando minimizar os erros nas conclusões. A estratégia de identificação de estudo, os critérios de inclusão e exclusão e as variáveis a serem consideradas são escolhidas de modo que outros 
investigadores possam chegar às mesmas conclusões (CORDEIRO et al., 2007; OLIVEIRA et al., 2010).

O presente estudo teve como objetivo executar uma revisão sistemática da literatura sobre T. minuta com o intuito de identificar os seus principais componentes químicos, usos populares e atividades biológicas.

\section{METODOLOGIA}

O método adotado foi uma revisão sistemática da literatura, conduzido de abril a maio de 2011. Na estratégia foi utilizada a palavra-chave "Tagetes minuta" e realizada busca no Scielo, Pubmed e Google acadêmico. O Google acadêmico foi utilizado por possuir publicações não indexadas, como teses, dissertações e trabalhos apresentados em congressos científicos, com objetivos de recuperar conhecimentos de circulação local ou regional, sendo pesquisadas publicações em idioma português e/ou espanhol.

Todos os artigos publicados em qualquer data que descreveram a composição química, o uso popular e atividade biológica foram utilizados como critério de inclusão. Foram excluídas publicações em que a partir do resumo, era identificado que não tratava dos temas escolhidos ou que as palavras-chave utilizadas para a pesquisa apareciam somente na discussão do artigo.

\section{Tagetes minuta}

$\mathrm{Na}$ pesquisa inicial foram identificadas 429 publicações. No Google acadêmico foram encontradas 384, no Scielo 9 e no Pubmed 36. À leitura do título e do resumo, das 384 publicações encontradas no Google acadêmico, foram incluídas 85, sendo que destas, 73 artigos foram obtidos integralmente e 12 foram obtidos somente o resumo. Os restantes 299 foram excluídos por não se adequarem aos critérios de inclusão ou serem repetidos. No Scielo, foram encontrados 9 artigos, 7 foram incluídos e 2 excluídos pelos mesmos critérios. No Pubmed foram encontrados 36, incluídos 19, sendo que destes, de 6 obteve-se somente os resumos e 13 foram obtidos integralmente, 17 foram excluídos.

Após este procedimento, foi realizada a leitura da publicação, e se estes se enquadravam dentro dos critérios de inclusão, a publicação era selecionada. Com este procedimento 
foram excluídos 60 artigos. Com esta metodologia foram selecionadas 51 publicações, sendo que de 6 foram lidos somente os resumos.

\section{Composição química}

Neste estudo, em todas as análises de composição química foi utilizado o óleo essencial de T. minuta. Os componentes químicos encontrados estão listados na Tabela 1 . Dos 13 compostos identificados, nenhum foi citado por todos os autores. O limoneno foi citado por 3 autores e 9 compostos foram citados por somente um autor. Chamorro et al. (2008) não especificaram a porcentagem dos componentes encontrados e Furtado et al. (2005) citaram apenas o principal composto químico da planta.

Tabela 1 - Principais compostos químicos encontrados na Tagetes minuta L.

\begin{tabular}{|c|c|c|c|c|}
\hline Composto químico & Ruffinengo et al. & Chamorro et al. & Gillij et al. & Furtado et al. \\
\hline$\alpha$-pineno & & & $11.8 \%$ & \\
\hline$\beta$-felandreno & & $x$ & & \\
\hline$\beta$ - ocimeno & & $x$ & & \\
\hline$\beta$-Bourbonene & & & $66.3 \%$ & \\
\hline Calameneno & & & $11.8 \%$ & \\
\hline Carvona & & & $0,1 \%$ & \\
\hline Di-idrotagetone & & $x$ & & $91,5 \%$ \\
\hline (E)-Ocimeno & $62.8 \%$ & & & \\
\hline (E)-Ocimenona & $6.6 \%$ & & $19.1 \%$ & \\
\hline Limoneno & $5.8 \%$ & $x$ & $66.3 \%$ & \\
\hline Tagetenone & & $x$ & & \\
\hline Tagetone & & $x$ & & \\
\hline (Z)-Ocimenona & $10.2 \%$ & & $2.7 \%$ & \\
\hline
\end{tabular}

Ruffinengo et al. (2007); Chamorro et al. (2008); Gillij et al. (2008); Furtado et al. (2005).

\section{Uso popular}

Os conhecimentos sobre as plantas medicinais no Rio Grande do Sul derivam dos índios, mas também dos colonizadores europeus que, ao aqui chegarem, procuraram, pelo método de tentativa e erro, encontrar plantas medicinais análogas às de seus países de origem. Esses 
conhecimentos foram transmitidos geralmente de forma oral, quase não existindo registros escritos sobre os mesmos (MENTZ et al., 1997).

Nesta revisão, foram encontradas informações a respeito do conhecimento sobre o uso popular de T. minuta em todas as regiões do Brasil, com exceção do centro-oeste.

Na região sul, foram encontradas seis publicações que falam sobre o resgate dos saberes tradicionais de $T$. minuta. $\mathrm{O}$ resgate de plantas com indicativo medicinal ou condimentar na região metropolitana de Porto Alegre citaram Tagetes minuta L. com o nome popular de "Chinchilho", "Picão-do-reino" e "Rojão" (WIEST et al., 2009a; WIEST et al., 2009b). Outros levantamentos etnobotânicos foram realizados próximos a Porto Alegre, em Viamão. Em um dos trabalhos, citava-se como nome popular o "Chinchilho", utilizando as folhas como repelente de pulga (HIDALGO, 2007) e houve um trabalho realizado em aldeia indígena Mbyá Guarani indicava o uso da decocção das folhas de "Chinchilla" para distúrbios digestivos com sintomas dolorosos intensos e presença de sangue nas fezes e recidivas de verminoses (HENNES, 2006). Em São Luiz Gonzaga, foi citado o seu uso para problemas estomacais e pneumonia, utilizando infusão e decocção das folhas de "picão-do-reino" (BARROS et al., 2007). Na região de Bandeirante, no Paraná, as folhas de "cravo de defunto" foram indicadas como depurativo do sangue, pele e ferida brava (FUCK et al., 2005).

Em Minas Gerais, foi feito um levantamento etnobotânico nas comunidades de Senador Firmino e foi indicado as flores e folhas de "cravo-de-defunto" contra problemas menstruais e verminose (KFFURI, 2008).

Na região norte, no município de Igarapé Mirí no Pará, foi indicando o chá e o sumo das folhas de "cravo" para febre, dor de cabeça, "doença que pega no ar", derrame e convulsão (PINTO, 2008).

Na Paraíba, o estudo foi no Cariri paraibano, indicando para $T$. minuta o nome popular de "coari-bravo", "cravo-de-viúva" e "cravo-bravo" como sedativo, febrífugo, contra amenorréia e vermífugo (AGRA et al., 2007). Já no Piauí, o estudo foi na Barra Grande e no Morro da Mariana, citando o "cravo-de-anjo" como medicinal, embora sem especificar o uso (SOUZA, 2010). 
Existem relatos do estudo em outros países da América do Sul sobre a T. minuta, como a Argentina e Bolívia. Na Argentina, foi realizado um resgate das plantas utilizadas na medicina popular em São Luis e Missiones, citando como nome popular "chilchita", "chil-chil", "chinchilla", "manzanilla de lasierra" como aromático, digestivo, tônico amargo, inseticida e antiparasitário (DEL VITTO et al., 1997; AMAT; VAJIA, 1991). Nos municípios de Córdoba e Formosa também foi realizado este tipo de pesquisa, citando como nome popular o "suíco" indicado como antiparasitário, digestivo, diarréia, cefaléias, dores estomacais, emético e vermífugo (MARTINEZ, 2005; MARTINEZ; PLANCHUELO, 2003; SCARPA, 2002; TOLEDO et al., 2009; TOLEDO, 2010). Na Bolívia foi realizado um resgate dos Vale Secos de Cochabamba sendo indicado $T$. minuta contra doenças do sistema nervoso central, paralisia facial e anorexia (RIVERO et al., 2002).

No Quênia, país da África, foi relatado efeito repelente sobre Anopheles gambiae quando queimada a planta, não sendo informado se a planta era de crescimento espontâneo na região (SEYOUM et al., 2002b).

\section{Atividade biológica}

As plantas medicinais, após um período em que foram colocadas em segundo plano, em consequência do surgimento de novos medicamentos, voltam a atrair a atenção de pesquisadores, profissionais da saúde, bem como da população em geral, a qual busca atender a necessidade de recursos terapêuticos através da medicina popular. Frente a essa ampliação do uso, profissionais da saúde e pesquisadores se preocupam com o embasamento científico necessário para avaliar a eficácia e os possíveis riscos das plantas em uso (MENTZ et al., 1997). Alguns trabalhos sobre as atividades biológicas com fins medicinais de T. minuta estão resumidos na Tabela 2.

Tabela 2 - Tipo de extrato, atividade biológica, espécies e autor referencial.

\begin{tabular}{llll}
\hline \multicolumn{1}{c}{ Extrato } & \multicolumn{1}{c}{ Atividade } & Espécie & Referência \\
\hline Decocto & Antimicrobiano & Staphylococcus aureus, & SOUZA et al., 2000 \\
& & Enterococcus faecium, & \\
& & Salmonella gallinarum, & \\
& & Escherichia coli & \\
\hline Extrato metanólico & Antimicrobiano & Escherichia coli & VIEIRA et al., 2011 \\
\hline
\end{tabular}




\section{Candida kruzei}

$\begin{array}{llll}\text { Decocto e extrato Antimicrobiano } & \text { Staphylococcus agalactiae, } & \text { SCHUCH et al., } 2008\end{array}$

hidro alcóolico

Staphylococcus aureus

\begin{tabular}{|c|c|c|c|}
\hline Extrato & Antimicrobiano & $\begin{array}{l}\text { Gram-positivas } \\
\text { Gram-negativas }\end{array}$ & $\begin{array}{l}\text { TERESCHUK et al., } \\
1997\end{array}$ \\
\hline Extrato & Antimicrobiano & Paenibacillus larvae & $\begin{array}{l}\text { GONZÁLEZ ; } \\
\text { MARIOLI, } 2010\end{array}$ \\
\hline $\begin{array}{l}\text { Extrato aquoso } \\
\text { Extrato alcoólico }\end{array}$ & Antimicrobiano & Escherichia coli & WIEST et al., 2009a \\
\hline $\begin{array}{l}\text { Extrato aquoso } \\
\text { Extrato alcoólico }\end{array}$ & Antimicrobiano & Salmonella spp. & WIEST et al., 2009b \\
\hline Extrato metanólico & Antimicrobiano & $\begin{array}{l}\text { Fusarium proliferatum } \\
\text { Fusarium verticillioides }\end{array}$ & THEMBO et al., 2010 \\
\hline Óleo essencial & Antimicrobiano & $\begin{array}{l}\text { Trichophyton mentagrophytes, } \\
\text { Microsporum gypsum, } \\
\text { Candidaralbicans, } \\
\text { Cryptococcus neoformans, } \\
\text { Aspergillus niger e Penicillium } \\
\text { spp }\end{array}$ & Bll et al., 2000 \\
\hline Óleo essencial & Larvicida & Aedes aegypti & GREEN et al., 1991 \\
\hline Óleo essencial & Larvicida & Aedes aegypti & LIMA et al., 2009 \\
\hline Óleo essencial & Larvicida & Aedes aegypti & $\begin{array}{l}\text { FURTADO et al., } \\
2005\end{array}$ \\
\hline Extrato hexânico & Larvicida & Meloidogyneincognita & $\begin{array}{l}\text { SCRAMIM et al., } \\
1990\end{array}$ \\
\hline Óleo essencial & Repelente & Aedes aegypti & GILLIJ et al., 2008 \\
\hline Extrato & $\begin{array}{l}\text { Repelente, inseticida } \\
\text { e nematicida }\end{array}$ & $\mathrm{N} / \mathrm{I}$ & $\begin{array}{l}\text { NACHTIGAL; } \\
\text { SCHNEIDER, } 2007\end{array}$ \\
\hline Óleo essencial & Inseticida & Pediculus humanus capitis & CESTARI et al., 2004 \\
\hline Óleo essencial & Inseticida & Callosobruchusmaculatus & KÉİTA et al., 2000 \\
\hline
\end{tabular}




\begin{tabular}{llll}
\hline Extrato metanólico & Inseticida & Phlebotomus duboscqi & IRERI et al., 2010 \\
\hline Óleo essencial & Anti-parasitário & Ectoparasitas & FURTADO et al., \\
& & & 2010 \\
\hline Óleo essencial & Anti-parasitário & Varroa destructor & RUFFINENGO et al., \\
& & & 2007 \\
\hline N/l & Anti-parasitário & Endoparasitas & SIMS et al., 2000 \\
\hline Extrato aquoso & Nematicida & Meloido gyneincognita & JUNGES et al., 2009 \\
\hline Cultivo da planta & Nematicida & Meloido gynejavanica & MATSUMOTO et al., \\
& & & 2002
\end{tabular}

O decocto, extrato hidroalcoólico, extrato metanólico, extrato aquoso e óleo essencial da planta demonstraram atividade antibacteriana contra Staphylococcus aureus, Staphylococcus agalactiae, Enterococcus faecium, Salmonella spp., Salmonella gallinarum, Escherichia coli, Paenibacillus larvae (GONZÁLEZ; MARIOLI, 2010; SCHUCH et al., 2008; SOUZA et al., 2000; TERESCHUK et al., 1997; VIEIRA et al., 2011; WIEST et al., 2009a; WIEST et al., 2009b). Barbosa et al. (1994) afirmaram que o extrato fluido da planta possui pequena atividade antibacteriana, não sendo notada nenhuma atividade contra os bacilos gramnegativos.

O extrato metanólico demonstrou efeito antifúngico contra Candida krusei, Fusarium verticillioides, Fusarium proliferatum, Aspergillus flavus, Aspergillus parasiticus, Trichophyton mentagrophytes, Microsporum gypsum, Candida albicans, Cryptococcus neoformans, Aspergillus niger e Penicillium spp (BII et al., 2000; THEMBO et al., 2010; VIEIRA et al., 2011), atuando também no crescimento micelial de Colletrotrichum gloeosporioides e Botrytis cinerea. Já o extrato bruto utilizando água deionizada, promoveu o desenvolvimento de fungo e o crescimento micelial de Microsporum canis e Trichoderma sp. (CATARINO et al., 1988; FRIAS; KOZUSNY-ANDREANI, 2010).

O extrato etanólico e óleo essencial de T. minuta foram utilizados como larvicidas contra Aedes fluviatilis, Aedes aegypti e Meloido gyneincognita (FURTADO et al., 2005; GREEN et al., 1991; LIMA et al., 2009; MACEDO et al., 1997; SCRAMIN et al., 1990), e o óleo essencial como repelente de Aedes aegypti (GILLIJ et al., 2008; NACHTIGAL; SCHNEIDER, 2007). No 
entanto Seyoum et al. (2002a), não observaram efeito repelente sobre Anopheles gambiae quando o vaso com a planta foi colocado no ambiente.

O extrato e óleo essencial são usados como inseticida contra Pediculus humanus capitis, Callosobruchus maculatus e Phlebotomus duboscqi (CESTARI et al., 2004; KÉïTA et al., 2000; NACHTIGAL; SCHNEIDER, 2007, IRERI et al., 2010).

O óleo essencial e decocto foram utilizados como anti-parasitários contra Varroa destructor e Boophilus microplus e alguns endoparasitários que atacam animais (FURTADO et al., 2010; RUFFINENGO et al.,2007; SIMS et al., 2000). Moyo et al. (2009) afirmaram que o extrato de T. minuta não possui efeito antiparasitário.

Trabalhos também relataram que o extrato aquoso é nematicida em plantações de tomate (JUNGES et al., 2009; MATSUMOTO et al., 2002; NACHTIGAL; SCHNEIDER, 2007), porém, há trabalhos que afirmam que este extrato aumenta o número de ovos, tornando assim a planta como hospedeira de nematóide em plantações de café (AMARAL et al., 2009; PONTE et al., 1996). Navarro e Barriga (1970) citaram a planta como resistente aos nematóides.

Seis artigos mostraram o uso do extrato de T. minuta com outras finalidades. O extrato aquoso foi descrito com efeito anti-hiperglicêmico (RANILLA et al., 2010), e a infusão das folhas foi utilizada na redução de peso em ratos Wistar (MARTINEZ et al., 2007). Ainda, foi avaliada a toxicidade aguda via oral em ratos Wistar, sendo descrito como atóxico a uma concentração de $26 \mathrm{~mL}$ de extrato fluido de $T$. minuta por cada quilo de peso corporal (JACOBUS et al., 2006). Toda planta, inclusive as flores, mostrou atividade contra carcinoma de pulmão (ICKES et al., 1973). Marotti et al. (2010) observaram sua atividade biocida em sistema de manejo integrado de pragas, enquanto Zydadlo et al. (1995) demonstraram o efeito antioxidante do óleo essencial.

\section{CONCLUSÃO}

A T. minuta é conhecida há alguns anos em várias regiões da América do Sul e vem sendo utilizada popularmente por suas atividades medicinais. Muitos trabalhos comprovam suas utilidades e bioatividades: medicinal, condimentar, aromática, inseticida, larvicida, nematicida, antiparasitária, repelente, antimicrobiana, anti-hiperglicêmica, biocida e 
antioxidante. O potencial bioativo com fins medicinais deve ser explorado mais adequadamente a partir do conhecimento mais detalhado da sua composição química, possibilitando elucidar suas diversas bioatividades.

\section{A SYSTEMATIC REVIEW OF Tagetes minuta L. (Asteraceae): POPULAR USE, THE CHEMICAL COMPOSITION AND BIOLOGICAL ACTIVITY}

\section{ABSTRACT}

$\mathrm{T}$ agetes minuta $\mathrm{L}$. is an aromatic plant commonly known as southern cone marigold, stinking roger or black mint among others. The objective of this work was to perform a systematic review of Tagetes minuta in order to identify the main chemical components, popular uses and biological activities. As a search strategy of the systematic review, the keyword "Tagetes minuta" was used in Scielo, Pubmed and Scholar Google. In the initial survey, 429 publications were found, 384 on Scholar Google, 9 on SciELO and 36 on Pubmed. After reading the title and abstract, 111 articles were evaluated since they fitted into the inclusion criteria. After this first step, the reading of the publication was carried out and whether they met the objectives of the work the publication would be selected. With this procedure, 51 articles were included. The systematic review showed that the plant Tagetes minuta, besides being popularly known for medical indications, was scientifically tested and showed antibacterial, antifungal, larvicide, insecticide, antiparasitic, nematicide, anti-hyperglycemia, antitumor, biocide and antioxidant effect.

Keywords: Tagetes minuta L. Systematic review. Bioactiveplant.

\section{REVISIÓN SISTEMÁTICA DE Tagetes minuta L. (Asteraceae): USO POPULAR, COMPOSICIÓN QUÍMICA Y ACTIVIDAD BIOLÓGICA}

\section{RESUMEN}

T agetes minuta L. es una planta aromática conocida comúnmente como Chinchilho, caléndula, chinchilla, entre otros. El objetivo de este trabajo fue realizar una revisión sistemática de Tagetes minuta con el objetivo de identificar los principales componentes químicos, utilización popular y actividades biológicas. Como estrategia de búsqueda de la revisión sistemática, se utilizo la palabra clave "Tagetes minuta" en el Scielo, Pubmed y Google Scholar. En la encuesta inicial 429 publicaciones fueran identificadas, en 
Google Académico 384, en SciELO 9 y 36 artículos de Pubmed. Después de leer el título y el resumen, se mantuvieron 111 títulos, ya que encajaban dentro de los criterios de inclusión. Después de este primer paso, la lectura de la publicación se llevó a cabo y si cumplían con los objetivos de la obra, era seleccionada la publicación. Con este procedimiento, se incluyeron 51 artículos. Esta revisión sistemática mostró que la planta Tagetes minuta es popularmente conocida por sus indicaciones médicas, ha sido probada científicamente y mostró actividad antibacteriana, antifúngica, larvicida, insecticida, antiparasitaria, nematicida, antihiperglicimia, antitumoral, biocida y antioxidante.

Palabras clave: Tagetes minuta L. Revisión sistemática. Plantas bioactivas.

\section{REFERÊNCIAS}

AGRA, M. F.; BARACHO, G. S.; BASÍLIO, I. J. D.; et al. Sinopse da flora medicinal do Cariri paraibano. Oecologia Brasiliensis, v. 11, n. 3, p. 323-330, 2007.

AMARAL, D. R.; OLIVEIRA, D. F.; CAMPOS, V. P.; et al. Effect of plant and fungous metabolites on Meloidogyne exigua. Ciência e Agrotecnologia, v. 33, p. 1861-1865, 2009.

AMAT, A. G.; VAJIA, M. E. Plantas Medicinales y Etnofarmacología en la Provincia de Misiones (Argentina). Acta Farmacéutica Bonaerense, v. 10, n. 3, p. 153-159, 1991.

BARBOSA, A. D.; FERREIRA, R. C. V.; VALENTE, P. H. M. Atividade antimicrobiana de extratos fluidos de plantas medicinais brasileiras. Lecta-USF, v. 12, n. 2, p. 153-163, jul.-dez. 1994.

BARROS, F. M. C.; PEREIRA, K. N.; ZANETTI, G. D.; et al. Plantas de Uso Medicinal no Município de São Luiz Gonzaga, RS, Brasil. Latin American Journal of Pharmacy, v. 26, n. 5, p. 652-662, 2007.

BELTRÁN, M. C. A. Etnobotánica de Plantas Vasculares en el Centro Poblado Llachón, Distrito Capachica, Departamento Puno, 2007-2008. Arequipa: UNSAA, 2008, 188p. Tesis (Biólogo), Facultad de Ciencias Biológicas y Agropecuarias, Universidad Nacional de San Agustín de Arequipa, 2008.

BII, C. C.; SIBOE, G. M.; MIBEY, R. K. Plant essential oils with promising antifungal activity. East African Medical Journal, v. 77, n. 6, p. 319-322, 2000.

CATARINO, V. M.; GHINI, R.; BETTIOL, W.; et al. Influência de extratos de folhas de Tagetes minuta e Vernonia polyanthes no crescimento micelial de Colletrotrichum gloeosporioides, Botrytis cinerea e Trichoderma sp. Summa Phytopathologica, v. 14, n. 1/2, p. 43, jan./jun. 1988. 
CESTARI, I. M.; SARTI, S. J.; WAIB, C. M.; et al. Evaluation of the potential inseticide activity of Tagetes minuta (Asteraceae) essencial oil against the head lice Pediculus humanus capitis (Phthiraptera: Pediculidae). Neotropical Entomology, v. 33, n. 6, nov./dez. 2004.

CHAMORRO, E. R.; BALLERINI, G.; SEQUEIRA, A. F.; et al. Chemical composition of essential oil from Tagetes minuta $\mathrm{L}$. leaves and flowers. Journal of the Argentine Chemical Society, $\mathrm{v}$. 96, n. 1-2, p. 80-86, 2008.

CORDEIRO, A. M.; OLIVEIRA, G. M.; RENTERÍA, J. M.; et al. Revisão sistemática: uma revisão narrativa. Revista do Colégio Brasileiro de Cirurgiões, v. 34, n. 6, p. 428-431, nov./dez. 2007.

DEL VITTO, L. A.; PETENATTI, E. M.; PETENATTI, M. E. Recursos herbolarios de San Luis (República Argentina) primeira parte: plantas nativas. Multequina, v. 6, p. 49-66, 1997.

FERRAZ, S.; FREITAS, L. H. O controle de fitonematóides por plantas antagonistas e produtos naturais. Departamento de Fitopatologia UFV, p. 1-17, 2008. Disponível em:

http://www.jcofertilizantes.com.br/pesquisa/pesquisa16-o-controle-de-fitonematoides.pdf. Acesso em: 25 de abr. 2011.

FRIAS, F. R.; KOZUSNY-ANDREANI, D. I. Utilização de extratos de plantas medicinais e óleo de Eucaliptus no controle in vitro de Microsporum canis. Revista Cubana de Plantas Medicinais, v. 15, n. 3, p. 119-112, 2010.

FUCK, S. B.; ATHANÁZIO, J. B.; LIMA, C. B.; et al.Plantas medicinais utilizadas na medicina popular por moradores da área urbana de Bandeirantes, PR, Brasil. Semina: Ciências Agrárias, v. 26, n. 3, p. 291-296, 2005.

FURTADO, F. N.; SILVA, V. A. R.; PEREIRA, J. R.; et al. Atividade carrapaticida do óleo essencial de Tagetes minuta. 2010. Disponível em:

http://revistas.ung.br/index.php/saude/article/view/688/777. Acesso em: 02 de maio 2011.

FURTADO, R. F.; LIMA, M. G. A.; NETO, M. A.; et al. Atividade larvicida de óleos essenciais contra Aedes aegypti L. (Diptera: Culicidae). Neotropical Entomology, v. 34, n. 5, p. 843-847, 2005.

GILLIJ, Y. G.; GLEISER, R. M.; ZYGADLO, J. A. Mosquito repellent activity of essential oils of aromatic plants growing in Argentina. Bioresource Technology, v. 99, n. 7, p. 2507-2515, 2008.

GONZÁLEZ, M. J.; MARIOLI, J. M. Antibacterial activity of water extracts and essential oils of various aromatic plants against Paeni bacillus larvae, the causative agent of American Foulbrood. Journal of Invertebrate Pathology, v. 104, n. 3, p, 209-213, 2010. 
GREEN, M. M.; SINGER, J. M.; SUTHERLAND, D. J.; et al. Larvicidal activity of Tagetes minuta (marigold) toward Aedes aegypti. Journal of the American Mosquito Control Association, $\mathrm{v}$. 7, n. 2, p. 282-286, 1991.

HATTORI, E. K. O. Asteraceae da estação ecológica do Panga, Uberlândia, Minas Gerais. Uberlândia: UFU, 2009. 169p. Dissertação (Mestrado em Ecologia e Conservação de Recursos Naturais), Instituto de Biologia, Universidade Federal de Uberlândia, 2009.

HENNES, M. Plantas medicinais nativas resgate da terapia indígena dos mbyá guarani. 2006. Disponível em:

http://www.plantasmedicinales.org/archivos/plantas_medicinales_empleadas_por_los_guar anies.pdf. Acesso em: 16 de abr. 2011.

HERRERA, B. L. Contribuición al estúdio florístico de La província de Concepción, (Junín): Dicotiledóneas. Lima: UNMSM, 2002. 117p. Tesis (Magister em Botânica Tropical), Universidad Nacional Mayor de San Marcos, 2002.

HIDALGO, D. E. Processo de transição na criação animal agroecológica no assentamento de reforma agrária: filhos de Sepé/Viamão-RS: manejo e sanidade. Florianópolis: UFSC, 2007. 73p. TCC (Graduação em Agronomia), Centro de Ciências Agrárias, Universidade Federal de Santa Catarina, 2007.

ICKES, G. R.; FONG, H. H.; SCHIFF Jr., P. L.; et al. Antitumor activity and preliminary phytochemical examination of Tagetes minuta (Compositae). Journal of Pharmaceutical Sciences, v. 62, n. 6, p. 1009-1011, 1973.

IRERI, L. N.; KONGORO, J.; NGURE, P.; et al. The potential of the extracts of Tagetes minuta Linnaeus (Asteraceae), Acalypha fruticosa Forssk (Euphorbiaceae) and Tarchonanthus camphoratus L. (Compositae) against Phlebotomus duboscqi Neveu Lemaire (Diptera: Psychodidae), the vector for Leishmania major Yakimoff and Schokhor. Journal of Vector Borne Diseases, v. 47, n. 3, p. 168-174, 2010.

JACOBUS, D.; MELLO, F. B.; MELLO, J. R. B. Doses repetidas de fitoterápico contendo Roripa nasturtium rusby, musa spp., Ficus carica linné, Tagetes minuta linné e mel (Xarope de agrião composto cibecol ${ }^{\circledR}$ ) Em ratos Wistar. Acta Scientiae Veterinariae, v. 34, n. 3, p. 295300, 2006.

JUNGES, E.; MORENO, M.; LIMA, D.; et al. Efeito do Extrato Aquoso e do Óleo Essencial de Tagetes minuta Aplicados ao solo sobre a Penetração de J2 de Meloidogyne incognita em Tomateiros. Revista Brasileira de Agroecologia, v. 4, n. 2, p. 1027-1030, 2009.

KÉİTA, S. M.; VICENT, C.; SCHMIT, J. P.; et al. Effect of various essential oils on Callosobruchus maculatus (F.) (Coleoptera: Bruchidae). Journal of Stored Products Research, v. 36, n. 4, p. 355-364, 2000. 
KFFURI, C. W. Etnobotânica de plantas medicinais no município de Senador Firmino (Minas Gerais). Viçosa: UFV, 2008. 101p. Dissertação (Mestrado em Magister Scientiae), Programa de Pós-Graduação em Fitotecnia, Universidade Federal de Viçosa, 2008.

KRUG, C. A comunidade de abelhas (Hymenoptera. Apiformes) da mata com araucária em Porto União-SC e abelhas visitantes florais da aboboreira (Cucurbita L.) em Santa Catarina, com notas sobre Peponapis fervens (Eucerini, Apidae). Criciúma: Unesc, 2007. 127p. Dissertação (Mestrado em Ciências Ambientais), Programa de Pós-Graduação em Ciências Ambientais, Universidade do Extremo Sul Catarinense, 2007.

LIMA, W. P.; NETO, F. C.; MACORIS, M. L. G.; et al. Estabelecimento de metodologia para alimentação de Aedes aegypti (Diptera-Culicidae) em camundongos swiss e avaliação da toxicidade e do efeito residual do óleo essencial de Tagetes minuta $\mathrm{L}$ (Asteraceae) em populações de Aedes aegypti. Revista da Sociedade Brasileira de Medicina Tropical, v. 42, n. 6, p. 638-641,2009.

LORENZI, H.; MATOS, F. J. A. Plantas medicinais do Brasil: nativas e exóticas. 2. ed. Nova Odessa: Instituto Plantarum, 2008. 544p.

MACEDO, M. E.; CONSOLI, R. A. G. B.; GRANDI, T. S. M.; et al. Screening of Asteraceae (Compositae) plants extracts for larvicidal activity against Aedes fluviatilis (Diptera: Culicidae). Memórias do Instituto Oswaldo Cruz, v. 92, n. 4, p.565-570, 1997.

MAROTTI, I.; MAROTTI, M.; PICCAGLIA. R.; et al. Thiophene occurrence in different Tagetes species: agricultural biomasses as sources of biocidal substances. Journal of the Science of Food and Agriculture, v. 90, n. 7, p. 1210-1217, 2010.

MARTINEZ, F. E. A.; IRUZUBIETA, L.; RODRIGUES, C.; et al. Efecto de Tagetes minuta L. sobre el peso corporal de ratas obesas. Boletín Latinoamericano y del Caribe de Plantas Medicinales y Aromáticas, v. 6, n. 6, p. 325-327, 2007.

MARTINEZ, G. J.; PLANCHUELO, A. M. La medicina tradicional de los Criollos Campesinos de Paravachasca y Calamuchita, Córdoba, Argentina. Scripta Ethnologica, v. 15, n. 25, p. 83-116, 2003.

MARTINEZ, G. J. Recolección y Comercialización de Plantas Medicinales en el Departamento Santa María, Provincia de Córdoba, Argentina. Acta Farmacéutica Bonaerense, v. 24, n. 4, p. 575-584, 2005.

MATSUMOTO, M. N.; GOULART, A. M. C.; HOMECHIM, M.; et al. Mulching with Pennisetum purpureum and other cultural practices for management of Meloidogyne javanica on tomato under greenhouse conditions. Nematologia Brasileira, v. 26, n. 1, p. 101-104, 2002.

MENTZ, L. A.; LUTZEMBERGER, L. C.; SCHENKEL, E. P. Da flora medicinal do Rio Grande do Sul: notas sobre a obra de D'Ávila (1910). Caderno de Farmácia, v. 13, n. 1, p. 25-48, 1997. 
MOYO, B.; MASIKA, P. J.; DUBE, S.; et al. An in-vivo study of the efficacy and safety of ethnoveterinary remedies used to control cattle ticks by rural farmers in the Eastern Cape Province of South Africa. Tropical Animal Health and Production, v. 41, n. 7, p. 1569-1576, 2009.

NACHTIGAL, J. C.; SCHNEIDER, E. P. Recomendações para produção de videiras em sistemas de bases ecológicas. 2007. Disponível em:

http://ainfo.cnptia.embrapa.br/digital/bitstream/CNPUV/9578/1/doc065.pdf. Acesso em: 10 de maio 2011.

NAVARRO, A. R.; BARRIGA, O. R. Control de nemátodos fitoparásitos por medio de rotación con cultivos resistentes a estos organismos. Revista ICA, v. 5, n. 3, p. 173-184, 1970.

OLIVEIRA, G. M.; CAMARGO, F. T.; GOLÇALVES, E. C.; et al. Revisão sistemática da acurácia dos testes diagnósticos: uma revisão narrativa. Revista do Colégio Brasileiro de Cirurgiões, v. 37, n. 2, p. 153-156, 2010.

PARRAS, J. M. M.; MOLERO, J.; CHUECA, F. E. Notas sobre la flora de la província de Granado. Lagascalia, v. 9, n. 1, p. 51-64, 1979.

PINTO, L. M. Plantas medicinais utilizadas em comunidades do município de Igarapé Miri, Pará: etnofarmácia do município de Igarapé Miri - PA. Belém: UFP, 2008. 98p. Dissertação (Mestrado em Ciências Farmacêuticas), Faculdade de Farmácia, Universidade Federal do Pará, 2008.

PONTE, J. J.; HOLANDA, Y. C. A.; ARAGÃO, M. L. Adendo ao catálogo de plantas hospedeiras de Meloidogyne no Brasil. Nematologia Brasileira, v. 20, n. 1, p. 73-81, 1996.

RANILLA, L. G.; KWON, Y.; APOSTOLIDIS, E.; et al. Phenolic compounds, antioxidant activity and in vitro inhibitory potential against key enzymes relevant for hyperglycemia and hypertension of commonly used medicinal plants, herbs and spices in Latin America. Bioresource Technology, v. 101, n. 12, p. 4676-4689, 2010.

RIVERO, S. A.; ATAHUACHI, M.; SARAVIA, E.; et al. Diversidad florística medicinal y potencial etnofarmacológico de las plantas de los Valles Vecos de Cochabamba-Bolivia. Revista Boliviana de Ecología y Conservación Ambiental, v. 12, p. 53-85, 2002.

ROCA, L. B.; GUZMÁN, B. H.; GÓMEZ, A. M. B.; et al. Caracterización física y tamizaje fitoquímico de la especie Tagetes erecta Lin. Revista Cubana de Química, v. 21, n. 2, p. 1015, 2009.

RUFFINENGO, S.; MAGGI, M.; FAVERIN, C.; et al. Essential oils toxicity related to Varroa destructor and Apis mellifera under laboratory conditions. Zootecnia Tropical, v. 25, n. 1. p. 63-69, 2007. 
SCARPA, G. F. Plantas empleadas contra trastornos digestivos en la Medicina Tradicional criolla del Chaco Noroccidental. 2002. Disponível em:

http://www.plantasmedicinales.org/archivos/plantas_medicinales_en_formosa.pdf. Acesso em: 3 de maio 2011.

SCRAMIN, S.; ZAVATTI, L. M. S.; SILVA, H. P.; et al. Utilização de extratos vegetais no controle de dois nematóides fitopatogênicos-Meloidogyne incognita e Tylenchulus semipenetrans.

EMBRAPA-CNPDA, Documentos 16, p. 46, 1990.

SCHUCH, L. F. D.; WIEST, J. M.; COIMBRA, H. S.; et al. Cinética da atividade antibacteriana in vitro de extratos naturais frente a microrganismos relacionados à mastite bovina. Ciência Animal Brasileira, v. 9, n. 1, p. 161-169, 2008.

SENATORE, F.; NAPOLITANO, F.; MOHAMED, M. A-H.; et al. Antibacterial activity of Tagetes minuta L. (Asteraceae) essential oil with different chemical composition. Flavour and Fragrance Journal, v. 19, n. 6, p. 574-578, 2004.

SEYOUM, A.; KABIRU, E. W.; LWANDE, W.; et al. Repellency of live potted plants against Anopheles gambiae from human baits in semi-field experimental huts. The American Journal of Tropical Medicine and Hygiene, v. 67, n. 2, p. 191-195, 2002a.

SEYOUM, A.; PALSSON, K.; KUNG'A, S.; et al. Traditional use of mosquito-repellent plants in western Kenya and their evaluation in semi-field experimental huts against Anopheles gambiae: ethnobotanical studies and application by thermal expulsion and direct burning. Transactions of The Royal Society of Tropical Medicine and Hygiene, v. 96, n. 3, p. 225-231, 2002b.

SIMS, B.; DIJKMAN, J.; ZAMBRANA, L. Logros del proyecto de mejoramiento de traccion animal 1997-2000. Cochabamba, Bolívia. II Seminário-Tallerde PROMETA, UMSS. p. 13-38, 2000.

SOUZA, C. A. S.; AVANCINI, C. A. M.; WIEST, J. M. Atividade antimicrobiana de Tagetes minuta L.-Compositae (Chinchilho) frente a bactérias Gram-positivas e Gram-negativas. Brazilian Journal of Veterinary Research and Animal Science, v. 37, n. 6, p. 1-9, 2000.

SOUZA, R. S. Etnobotânica e etnozoologia de comunidades pesqueiras da área de proteção ambiental (APA) do delta do Parnaíba, nordeste do Brasil. Teresina: UFPI, 2010. 176p. Dissertação (Mestrado em Desenvolvimento e Meio Ambiente), Universidade Federal do Piauí, 2010.

TERESCHUK, M. L.; RIERA, M. V. Q.; CASTRO, G. R.; et al. Antimicrobial activity of flavonoids from leaves of Tagetes minuta. Journal of Ethnopharmacology, v. 56, n. 3, p. 227-232, 1997. 
THEMBO, K. M.; VISMER, H. F.; NYAZEMA, N. Z.; et al. Antifungal activity of four weedy plant extracts against selected mycotoxigenic fungi. Journal of Applied Microbiology, v. 109, n. 4, p. 1479-1486, 2010.

TOLEDO, B. A. Diversidad de usos, prácticas de recolección y diferencias según género y edad en el uso de plantas medicinales en Córdoba, Argentina. Boletín Latinoamericano y del Caribe de Plantas Medicinales y Aromáticas, v. 8, n. 5, p. 389-401, 2009.

TOLEDO, B. A.; TRILLO, C.; GRILLI, M. Uso de plantas medicinales en relación al estado de conservación del bosque en Córdoba, Argentina. Ecología Austral, v. 20, p. 235-246, 2010.

VIEIRA, L. R. C.; NETO, A. G.; GONÇALVES, J. M.; et al. Avaliação da atividade antimicrobiana de Blainvillea biaristata DC. Tagetes minuta L. e Gochnatia oligocephala (Gardner) Cabrera. 2011. Disponível em: http://www.plamevasf.com/uploads/7/8/9/0/7890742/_resumo.444114-2011114.pdf. Acesso em: 20 de abr. 2011.

VISINTIN, A. M.; BERNARDELLO, G. Morfología y Anatomía floral de Tagetes minuta L. (Asteraceae). Arnaldoa, v. 12, n. 1-2, p. 8-15, 2005.

WIEST, J. M.; CARVALHO, H. H. C.; AVANCINI, C. A. M.; et al. Inibição e inativação de Escherichia coli por extratos de plantas com indicativo etnográfico medicinal ou condimentar. Food Science and Technology, v. 29, n. 3, p. 474-480, 2009 a.

WIEST, J. M.; CARVALHO, H. H. C.; AVANCINI, C. A. M.; et al. Inibição e inativação in vitro de Salmonella spp. com extratos de plantas com indicativo etnográfico medicinal ou condimentar. Arquivo Brasileiro de Medicina Veterinária e Zootecnia, v. 61, n. 1, p. 119127, 2009b.

ZYDADLO, J. A.; LAMARQUE, A. L.; MAESTRI, D. M. Use of essential oils as natural Antioxidants. Grasas y Aceites, v. 46, n. 4-5, p. 285-288, 1995. 